\title{
Finance Journals: Características dos Principais Periódicos, Autores Importantes e Artigos mais Citados
}

\author{
Flávia Cruz de Souza* \\ José Alonso Borba** \\ Newton Carneiro Affonso da Costa Júnior**** \\ Fernando Dal-Ri Murcia****
}

\begin{abstract}
Resumo
Este artigo teve como objetivo caracterizar os periódicos internacionais de Finanças disponibilizados pelo Portal Periódicos CAPES e conhecer os autores mais importantes da área. Após a exclusão de certos periódicos que não atendiam à metodologia proposta, 72 journals, que incluíam no seu título os termos finance e financial, foram analisados. Como resultados principais, destacam-se: Journal of Finance possui o maior fator de impacto (2,549); a maioria dos periódicos possui sede nos EUA $(70,83 \%)$ e publica quatro edições por ano; $51 \%$ dos periódicos cobram taxa de submissão, a qual varia de US\$ 40 a US\$ 450; grande parte dos periódicos leva em torno de seis meses para publicar um artigo já aprovado; o periódico Research in International Business and Finance possui o corpo editorial mais internacionalizado; o artigo mais citado foi publicado em 1997, no Journal of Finance; e Andrei Shleifer é autor dos três trabalhos mais citados no Scopus.
\end{abstract}

Palavras-chave: periódicos internacionais, artigos, autores, finance, financial.

Códigos JEL: G00.

Abstract

The objective of this paper is to characterize the Finance journals available in CAPES's Basis and to know the most important authors in this Area. After eliminating the publications that did not meet the proposed criterion, a total of 72 journals that contained the expression finance and financial in their titles have been analyzed. Main findings are: Journal of Finance has the highest impact factor $(2,549)$; the great majority of journals are NorthAmerican $(70,83 \%)$ and published four times a year; $51 \%$ of them charge a submission

Submetido em Novembro de 2007. Aceito em Abril de 2008. O artigo foi avaliado segundo o processo de duplo anonimato além se de ser avaliado pelos editores. Editor principal: Ricardo P. C. Leal. Os autores agradecem a CAPES e ao CNPq pelas bolsas de estudo concedidas aos autores, como também aos avaliadores da Revista Brasileira de Finanças pelas importantes considerações feita

*Mestranda em Administração - CPGA/Centro Sócio-Econômico. Universidade Federal de Santa Catarina, Campus Universitário, Trindade, Florianópolis - Santa Catarina - Brasil. CEP: 88040-900. Telefone: (48) 3721-9365. E-mail: flah@ flah.com.br

**Professor Adjunto do Departamento de Ciências Contábeis - CSE/UFSC e Professor Visitante da HEC-Montreal. E-mail: jalonso@cse.ufsc.br

****Professor Adjunto de Departamento de Ciências Econômicas - CSE/UFSC.

E-mail: newton@cse.ufsc.br

****Doutorando em Ciências Contábeis - FEA/USP. E-mail: fernandomurcia@hotmail.com 
fee that varies from US\$ 40 to US\$ 450; most part takes around six months to publish a paper once it gets accepted; Research in International Business and Finance has the most internationalized editorial board; the most cited article has been published in the Journal of Finance in the year of 1997; and Andrei Shleifer is the author of the three most cited papers in Scopus.

Keywords: journals, papers, authors, finance, financial.

\section{Introdução}

A publicação em periódicos é vista hoje como o meio mais adequado para o pesquisador expor suas idéias e descobertas, uma vez que revistas científicas são fontes de publicação permanentes. Os órgãos de fomento (CNPq, fundações estaduais de pesquisa) e as áreas de avaliação dos programas de pós-graduação da CAPES vêm incentivando a produção científica definitiva em periódicos de qualidade nacional e internacional. A publicação de estudos efetuados no Brasil em periódicos internacionais é fundamental para o reconhecimento da pesquisa e dos pesquisadores. Além de um periódico classificado pelo Qualis/CAPES como "Internacional A" pontuar duas vezes mais que um classificado como "Nacional A", ou seja, 24 pontos, uma pesquisa publicada nesses periódicos pode alavancar, disseminar e contribuir para o desenvolvimento da pesquisa realizada no país.

Em âmbito nacional, não possuímos uma grande variedade de periódicos específicos para as áreas da Administração como, por exemplo, Contabilidade, Recursos Humanos, Marketing, Finanças, mas sim periódicos gerais de Administração. Mais especificamente em Finanças, apesar de várias revistas de Administração (Revista de Administração de Empresas, Revista de Administração Contemporânea, Revista de Contabilidade e Finanças) publicarem artigos e existirem congressos (Encontro Brasileiro de Finanças, Encontro da ANPAD, Congresso USP de Controladoria e Contabilidade) para trabalhos dessa disciplina, existe no Brasil apenas um periódico específico - a Revista Brasileira de Finanças -, o qual é classificado como "Nacional A" na área de Administração, Ciências Contábeis e Turismo pelo Qualis/CAPES. Entretanto, internacionalmente, há muitas opções de journals para um pesquisador publicar seus trabalhos.

Em Finanças, a pesquisa científica teve início dando ênfase a normatização e padronização das demonstrações contábeis, com o intuito de comparar desempenhos empresariais. Essa fase inicial foi chamada de Finanças Antigas, a qual se baseava em Contabilidade e Direito. (Camargos et alii, 2005)

De acordo com Haugen (2000), as Finanças Modernas se baseiam em quatro pilares: uma ferramenta para composição de carteiras de ativos criada por Harry Markowitz; o modelo CAPM (Capital Asset Pricing Model); a contribuição de Modigliani e Miller (1958) relativa à estrutura de capital; e a proposição dos mercados eficientes de Fama (1970). O desenvolvimento das Finanças Modernas refletiu o ambiente econômico global, uma vez que seu foco reorientou-se para a análise teórica e para as decisões administrativas voltadas para a escolha de ativos e passivos para maximizar o valor da empresa. 
O objetivo principal deste artigo é realizar um levantamento acerca das características de Finance Journals, além de conhecer os autores e artigos mais citados da área, tanto pelo ISI/Thompson (Institute for Scientific Information) quanto pelo Scopus. Ressalta-se que este estudo é inédito no Brasil, uma vez que nenhum outro artigo publicado procurou conhecer e avaliar periódicos internacionais de Finanças.

De uma maneira geral, o presente artigo busca contribuir para a construção do conhecimento científico na área de Finanças, na medida em que identifica e segmenta as principais revistas acadêmicas da área. Neste sentido, o estudo oferece oportunidades para futuros autores interessados em desenvolver pesquisas e eventualmente publicar seus achados em veículos internacionais. Do mesmo modo, são apresentadas algumas das principais pesquisas já realizadas na área e seus respectivos autores. Além disso, os resultados podem fornecer insights para o desenvolvimento de novos estudos no cenário nacional.

Este artigo é composto de outras quatro seções, a saber: a seção 2 aborda a revisão teórica, onde são relatadas pesquisas anteriores e informações sobre o Portal CAPES e bases de dados do Scopus e do ISI; a seção 3 define os aspectos metodológicos; a seção 4 evidencia a análise dos resultados; e a seção 5 apresenta as considerações finais do estudo.

\section{Revisão Teórica}

\subsection{Pesquisas anteriores}

Muitos artigos já foram desenvolvidos com o objetivo de avaliar a pesquisa científica em Finanças, principalmente no exterior. Neuhauser (2007) debateu a questão da pesquisa survey na área, além de apresentar tendências de estudos futuros em Finanças. Baker e Mukherjee (2007) desenvolveram uma pesquisa entrevistando editores de journals da área, com o objetivo de demonstrar a importância das pesquisas do tipo survey. Em âmbito nacional, o principal estudo da área foi desenvolvido por Leal et alii (2003), os quais elaboraram um estudo bibliométrico com base em 551 artigos publicados em um evento e periódicos brasileiros.

Alguns estudos objetivaram analisar a quantidade de citações de artigos e autores, como é o caso do trabalho de Peng e Zhou (2006), os quais estudaram a área de Gestão Estratégica. Borokhovich et alii (2000) foram mais adiante e estudaram os três journals em Finanças de maior fator de impacto: Journal of Finance, Journal of Financial Economics e Review of Financial Studies. 
Também há artigos que realizaram estudos bibliométricos a partir de periódicos internacionais. Frezatti e Borba (2000) analisaram características de periódicos científicos internacionais da área de Contabilidade. Frandsen (2004), por exemplo, analisou journals da área de Economia e fez uma análise das citações a partir desses periódicos. Por outro lado, Chan et alii (2002) analisaram um journal específico da área de Finanças, o Pacific-Basin Finance Journal, com o objetivo de discutir os artigos publicados nos 10 anos do periódico, além de demonstrar características como impacto, autoria e pesquisas empíricas desses estudos.

Diversos outros estudos procuraram criar rankings a partir da produtividade científica. Grable (2006) classificou faculdades americanas quanto à sua publicação em periódicos da área de Finanças. Chan e Fok (2003) analisaram os conselhos editoriais de 60 journals de Finanças e concluíram que professores membros de conselhos editoriais influenciam na qualidade da pesquisa nas faculdades (departamentos) às quais estão vinculados. Chan et alii (2004) criaram um ranking de universidades européias com base na publicação em periódicos internacionais de Finanças na década de 90. Um ranking de periódicos internacionais da área contábil foi desenvolvido por Borba e Murcia (2006), os quais propuseram uma metodologia para avaliar journals.

\subsection{Portal CAPES, Scopus e ISI}

A grande quantidade de pesquisas desenvolvidas tem demandado sistemas que armazenem, gerenciem e disponibilizem aos usuários a informações de que este necessita. Como conseqüência desse volume produzido, ampliam-se os sistemas de recuperação e armazenamento de informação. A seguir, serão apresentadas as bases de dados do Portal Periódicos CAPES, Scopus e ISI.

\section{Portal CAPES}

Constituindo-se como uma das principais fontes de informação acadêmica, ao permitir acesso a grande parte da produção científica mundial, o Portal Periódicos CAPES reúne, em sua interface, um conjunto expressivo de periódicos nacionais e estrangeiros. São mais de 10.000 revistas e mais de 90 bases de dados referenciais que abrangem todas as áreas do conhecimento. Esta ferramenta é viabilizada pela Coordenação de Aperfeiçoamento de Pessoal de Nível Superior - CAPES -, e disponibilizada a instituições de ensino superior e de pesquisa.

\section{Scopus}

A Scopus é uma base de dados multidisciplinar, produzida pela editora Elsevier desde 2004, com cobertura desde 1960, que contém resumos de 27 milhões de artigos, referências e índices da literatura científica, técnica e médica. Pode incluir citações e links para texto completo de artigos, quando esses estão disponíveis. Segundo a empresa, a base de dados indexa 14.000 títulos de periódicos de 4.000 editoras internacionais, com atualizações diárias. Mais de $60 \%$ dos títulos são de outros países que não os Estados Unidos e $85 \%$ do conteúdo é indexado utilizando os 
vocabulários controlados para a definição das palavras-chave e/ou descritores. A base pesquisa, simultaneamente, o conteúdo dos artigos indexados em sua própria plataforma, na web e ainda bases de patentes sobre o assunto pesquisado. A base Scopus cobre as seguintes áreas do conhecimento: química, física, matemática, engenharia, ciências da saúde e vida, ciências sociais, psicologia, economia, biologia, agricultura, ciências ambientais e ciências gerais. (Mesquita, 2006)

\section{ISI (Institute for Scientific Information)}

A missão básica do Institute for Scientific Information (ISI), como uma companhia publicadora de bases de dados, é oferecer uma cobertura abrangente da mais importante e influente pesquisa realizada em todo o mundo. Atualmente, a base de dados ISI compreende mais de 16 mil títulos de revistas, livros e anais de congressos internacionais nas áreas de ciências, ciências sociais, artes e humanidades. Uma parte importante dessa base são os 8 mil títulos de revistas internacionais que o ISI indexa anualmente, registrando os dados bibliográficos completos para cada documento incorporado, incluindo os resumos originais em inglês, os endereços dos autores e editores e as referências bibliográficas citadas em cada revista. (Testa, 1998)

O ISI existe desde 1986 e usa dois critérios na escolha das revistas que compõem a sua base de dados: a periodicidade e o fator de impacto da revista, que é medido pelo número de citações de seus artigos em outras revistas. O ranking dos periódicos pelo fator de impacto é publicado pelo JCR (Journal Citation Reports), publicação anual criada em 1975, que oferece recursos para a avaliação dos títulos que compõem a base da Web of Science. Este fator de impacto é definido matematicamente como o número de vezes que os artigos das revistas são citados durante um período específico (o numerador), dividido pelo número total de artigos publicados por esta revista no mesmo período (denominador), num período convencional de dois anos. Não há como medir quanto um artigo foi utilizado pelos profissionais, mas pode-se medir seu efeito para outros pesquisadores e autores, examinando com que freqüência e onde foram citados em outros artigos. O JCR é importante ferramenta auxiliar tanto para o pesquisador, que poderá determinar onde deve publicar seus trabalhos, assim como para os bibliotecários realizarem análise de coleção de periódicos. (Vilhena e Crestana, 2002)

\section{Aspectos Metodológicos}

A amostra de periódicos foi coletada a partir do Portal Periódicos CAPES (www.periodicos.capes.gov.br), através da busca de dois termos: financial e $f$ nance. Quanto à palavra financial, foram encontrados 80 periódicos, e quanto à palavra finance foram encontrados 107 periódicos. 
Uma série de exclusões foi feita a partir desse ponto com o objetivo de se ter uma amostra adequada. Assim, começou-se pela exclusão de periódicos que são em outro idioma que não o inglês. Além disso, foram eliminados os que estavam duplicados, uma vez que o mesmo periódico pode estar hospedado em várias bases de dados diferentes (Blackwell, Science Direct, Proquest, dentre outros). Após esses cortes, ainda foram eliminados os periódicos que não tiveram edições publicadas pelas bases de dados até, pelo menos, dezembro de 2004. A quarta exclusão foi de periódicos que somente publicam artigos curtos, de 1 a 5 páginas, uma vez que o objetivo era de se chegar a uma amostra de periódicos que publicam artigos acadêmicos. Por fim, foram eliminados os periódicos que não utilizam o procedimento de blind review (revisão às cegas), pois a existência dessa característica demonstra, em tese, que o periódico é mais científico do que outros que não adotam esse procedimento. Assim, a amostra final totalizou 41 periódicos de finance e 31 periódicos de financial, totalizando 72 journals. A Tabela 1 resume essas etapas e apresenta a quantidade de periódicos eliminados em cada uma delas.

Tabela 1

Metodologia da exclusão de periódicos

\begin{tabular}{lcc}
\hline \multicolumn{1}{c}{ Etapa } & Finance & Financial \\
\hline Amostra inicial & 107 & 80 \\
Periódicos em língua francesa & 2 & 0 \\
Periódicos duplicados & 24 & 19 \\
Periódicos não-publicados até dezembro/2004 & 10 & 12 \\
Periódicos com artigos curtos (não-científicos) & 18 & 13 \\
Periódicos sem blind review (revisão às cegas) & 12 & 5 \\
Amostra final & 41 & 31 \\
\hline
\end{tabular}

Após essas exclusões, periódicos foram analisados de acordo com as seguintes questões:

- Se estavam indexados ao Scopus e ao ISI, além de apresentarem o "fator de impacto" calculado pelo JCR (Journal Citation Report) no site do periódico;

- Quais os países-sede dos periódicos. Característica obtida através do país da associação ou universidade ao qual o periódico estava vinculado. Quando não existia vinculação com alguma instituição, foi considerado o país da maioria dos editores avaliadores;

- Qual a periodicidade dos journals analisados;

- Se existe de taxa de submissão. Os periódicos que cobram uma taxa de submissão foram classificados através das seguintes categorias de intervalos: de US\$ 1 - US\$ 50, US\$ 51 - US\$100, US\$ 101 - US\$150, US\$ 151 - US\$ 200 e Acima de US\$200;

- Através de pesquisa enviada por e-mail aos editores-chefe foi obtida a taxa de corte e tempo para publicação do artigo após aprovação; 
- Cálculo do Índice de Internacionalização do Corpo Editorial (IICE), com o objetivo de determinar se os periódicos realmente possuem especialistas de vários países, independente da universidade em que cada um está vinculado. Este cálculo foi feito utilizando-se a quantidade de países representados no corpo editorial do periódico, a quantidade de editores estrangeiros (considerou-se o país do periódico) e a quantidade total de editores do periódico.

- E, por fim, são apresentados os autores e artigos mais citados que foram publicados nos periódicos analisados. Os autores da área de Finanças mais citados foram coletados no ISI e os artigos mais citados foram obtidos através do Scopus, utilizando-se como critério mais de 250 citações.

\section{Descrição e Análise dos Resultados}

Esta seção está dividida em 6 subseções, a saber: apresentação do total de periódicos analisados e indexados ao Scopus e/ou ISI, além do fator de impacto de alguns deles; países-sede dos periódicos; periodicidade dos journals; cobrança de taxa de submissão; taxa de corte, tempo para publicação e índice de internacionalização; e, autores e artigos mais citados.

\subsection{Periódicos analisados e indexação ao Scopus, ISI e JCR}

Os periódicos apresentados nos Apêndices 1 e 2 compõem a amostra final analisada. O Apêndice 1 apresenta somente os 49 periódicos que são indexados ao Scopus, os 25 indexados ao ISI e fator de impacto (JCR) de 18 deles, uma vez que poucos apresentam tal informação em sua página na Internet.

A partir do Apêndice 1, percebe-se que o Journal of Finance possui o maior grau de impacto $(2,549)$, ou seja, de acordo com o fator de impacto este é o principal periódico internacional da área e conseqüentemente, um dos mais criteriosos quanto à publicação. Em seguida, tem-se o Journal of Financial Economics, com fator de impacto de 2,385 e o Review of Financial Studies, com fator de impacto de 1,893 .

Sendo assim, todos os periódicos indexados ao ISI são considerados importantes e aqueles com fator de impacto superior a 0,5 são avaliados pela área como "Internacional A".

Por outro lado, periódicos como International Journal of Finance and Economics e Emerging Markets, Finance and Trade demonstraram pouca importância, uma vez que o fator de impacto foi menor que 0,3 .

Pode-se notar que grande parte dos periódicos que são indexados ao ISI, também o são ao Scopus, exceto o European Financial Management e o Managerial Finance. 
Já o Apêndice 2 demonstra os periódicos que não são indexados a nenhuma das bases de dados pesquisadas. Os 21 periódicos listados, apesar de não estarem indexados às bases de dados pesquisadas, também publicaram estudos relevantes para a área de Finanças.

Vale ressaltar que os periódicos apresentados no Apêndice 2, apesar de não estarem indexados ao Scopus ou ao ISI, foram analisados juntamente com os periódicos do Apêndice 1, totalizando 72 journals.

\subsection{Países dos periódicos}

A Figura 1 apresenta os países representados pelos periódicos. Como já mencionado no capítulo da metodologia, foi considerado como país do periódico o país da associação ou universidade que o apóia. Quando não existia alguma instituição vinculada ao periódico, o país da maioria dos editores avaliadores era considerado como país do periódico.

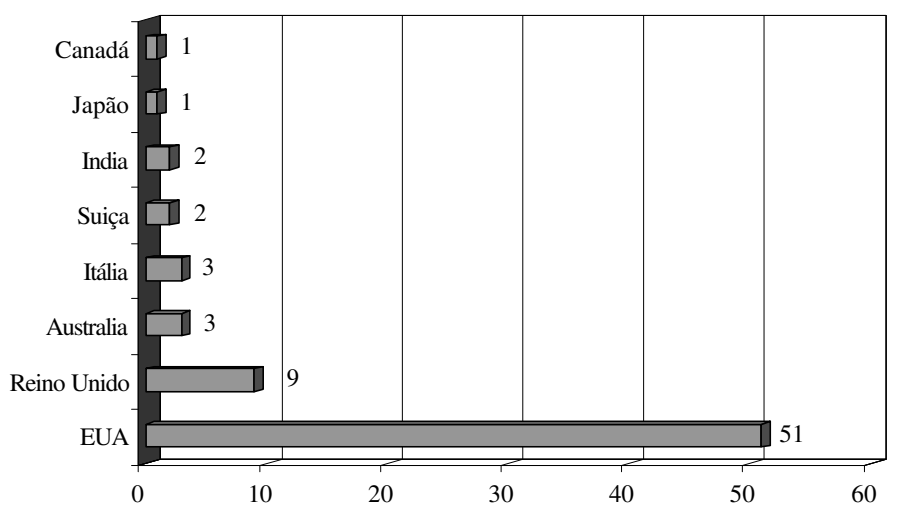

Fonte: dados da pesquisa

Figura 1

Países dos periódicos

Evidencia-se, através da Figura 1, que 70,83\% dos periódicos representam os Estados Unidos. Isso pode ser explicado pelo desenvolvimento da pesquisa em Finanças no país, além do crescente número de pesquisadores na área. Em segundo lugar, o Reino Unido foi classificado como país de 9 periódicos dos 72 da amostra. 


\subsection{Periodicidade}

Na Figura 2, são apresentadas as quantidades de edições publicadas por ano dos periódicos.

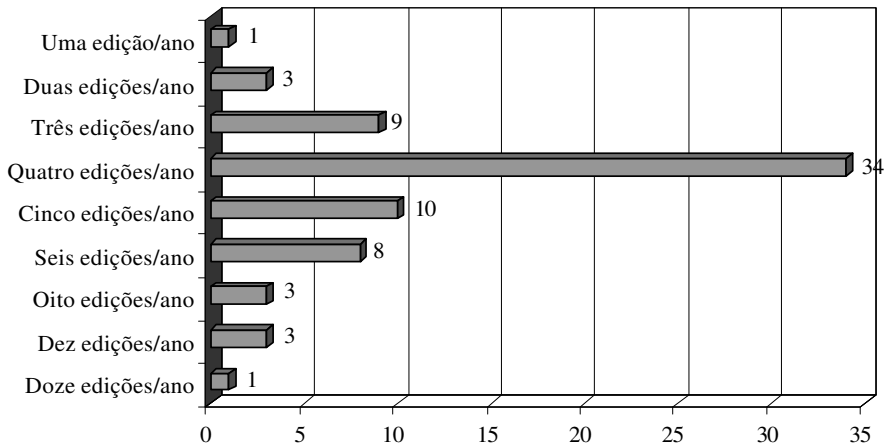

Fonte: Dados da pesquisa

Figura 2

Periodicidade dos journals

Percebe-se que grande parte dos periódicos é publicada trimestralmente, uma vez que $47,22 \%$ dos periódicos analisados possuem quatro edições por ano. Em seguida, há certa homogeneidade no que se refere à quantidade de periódicos que publicam três, cinco e seis edições por ano.

\subsection{Taxa de submissão}

A Figura 3 apresenta as porcentagens dos periódicos que cobram e dos que não cobram taxa de submissão.
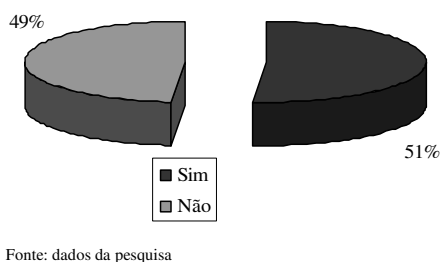

Figura 3

Existência de taxa de submissão

Na Figura 3, pode-se notar um equilíbrio no que se refere à cobrança de taxa de submissão, pois $51 \%$ dos periódicos a cobram e, conseqüentemente, $49 \%$ não a cobram. 
Enquanto que no Brasil os periódicos não cobram taxa de submissão, os principais journals somente aceitam submissões mediante o pagamento de uma taxa.

A partir dos periódicos que cobram taxa de submissão, foi feita uma nova classificação, utilizando-se de intervalos de taxas, apresentados na Figura 4.

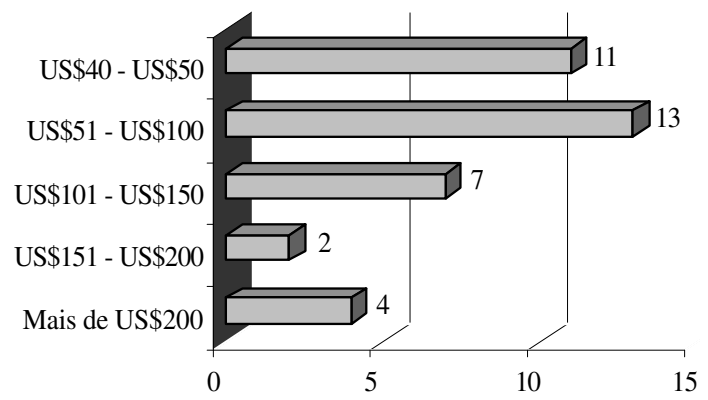

Fonte: dados da pesquisa

Figura 4

Taxa de submissão dos periódicos

Evidencia-se, a partir da Figura 4, que 13 periódicos cobram uma taxa de submissão entre US\$ 51 e US\$ 100. Em segundo lugar, 11 periódicos cobram uma taxa de US\$ 40 a US\$ 50. Assim, percebe-se que a maioria dos periódicos que cobram taxa de submissão, o fazem como sendo até US\$100.

Dentre os 37 periódicos que cobram taxa de submissão, o Finance India e o Quarterly Review of Economics and Finance cobram US\$ 40 por submissão, enquanto que o European Financial Management cobra US\$ 450 por submissão, respectivamente o menor e maior valor de taxas de submissão na amostra analisada.

Vale ressaltar que os valores de taxa de submissão coletados e analisados neste artigo são para pesquisadores que não assinam os periódicos. Grande parte dos journals cobra taxa de submissão menor para assinantes.

\subsection{Taxa de corte, tempo para publicação e IICE}

No Apêndice 3 são apresentadas as respostas dos editores-chefe com as respectivas taxas de corte e tempo para publicação após um artigo ser aprovado. Além dessas informações, é demonstrado o Índice de Internacionalização do Corpo Editorial (IICE), o qual foi calculado da seguinte maneira:

$$
I I C E_{i}=\sqrt{\frac{N P_{i} \times N E_{i}}{\left(N T A_{i}\right)^{2}}}
$$


onde:

$N P_{i}=$ número de países representados no corpo editorial do periódico (excluindo na contagem o país do periódico),

$N E_{i}=$ número de editores estrangeiros (considerou-se o país do periódico) e, $N T A_{i}=$ número total de editores do periódico.

A partir do Apêndice 3, pode-se notar que vários periódicos demoram em torno 6 meses para publicar um artigo já aprovado. Por outro lado, periódicos como Finance India, Journal of International Financial Markets, Institutions and Money e Journal of Multinational Financial Management demoram ao menos 15 meses para publicar um artigo já aprovado pelos avaliadores.

Quanto à taxa de corte, a maioria dos periódicos aprova $20 \%$ ou menos dos artigos submetidos a eles. Vale mencionar que o Journal of Corporate Finance possui taxa de corte de 95\%, o Journal of Finance, o Mathematical Finance e Review of Financial Studies possuem taxa de corte de 94\%. Entretanto, esses journals possuem baixo IICE.

Percebe-se, ainda, que o periódico Research in International Business and Finance $(0,685)$ possui o corpo editorial mais internacionalizado na amostra de periódicos analisada, seguido do International Journal of Health Care Finance and Economics $(0,666)$ e do Financial History Review $(0,562)$. Em tese, periódicos com esse perfil seriam mais receptivos à publicação de artigos de países que não utilizam o mesmo banco de dados do país-sede do journal.

Por outro lado, os periódicos Journal of Applied Finance, Journal of Economics and Finance, Journal of Financial and Quantitative Analysis, Journal of Financial Economics e Research in Healthcare Financial Management apresentaram IICE nulo, evidenciando a existência de editores de somente um país.

\subsection{Autores e artigos mais citados}

Na Tabela 2 são apresentados os artigos mais citados dentre os periódicos analisados. Essa coleta de dados foi feita através do Scopus, base de dados que demonstra os estudos mais citados durante os anos. O critério utilizado foi selecionar os artigos que possuíam mais de 250 citações até o final de 2006. 
Tabela 2

Artigos mais citados publicados nos periódicos analisado

\begin{tabular}{|c|c|c|c|c|}
\hline Citações & Periódico & Ano & Título & Autores \\
\hline 713 & Journal of Finance & 1997 & A survey of corporate governance & Shleifer, Andrei; Vishny, Robert W. \\
\hline 617 & Journal of Finance & 1997 & Legal determinants of external finance & La Porta, Rafael; Lopez-de-Silanes, \\
\hline 518 & Journal of Finance & 1999 & Corporate ownership around the world & $\begin{array}{l}\text { Florêncio; Shleifer, Andrei; Vishny, Robert W. } \\
\text { La Porta, Rafael; Lopez-de-Silanes, }\end{array}$ \\
\hline 437 & Journal of Finance & 1996 & $\begin{array}{l}\text { Multifactor explanations of asset pricing } \\
\text { anomalies }\end{array}$ & $\begin{array}{c}\text { Florêncio; Shleifer, Andrei } \\
\text { Fama, Eugene F.; French, Kenneth R. }\end{array}$ \\
\hline 417 & Journal of Finance & 1997 & $\begin{array}{l}\text { On persistence in mutual fund perfor- } \\
\text { mance }\end{array}$ & Carhart, Mark M. \\
\hline 342 & Journal of Financial Economics & 1998 & $\begin{array}{l}\text { Market efficiency, long-term returns, and } \\
\text { behavioral finance }\end{array}$ & Fama, Eugene $\mathrm{F}$. \\
\hline 337 & Mathematical Finance & 1999 & Coherent measures of risk & $\begin{array}{l}\text { Artzner, Philippe; Delbaen, Freddy; Eber, } \\
\text { Jean Marc; Heath, David }\end{array}$ \\
\hline 311 & Journal of Finance & 1998 & $\begin{array}{l}\text { Investor psychology and security market } \\
\text { under- and overreactions }\end{array}$ & $\begin{array}{l}\text { Daniel, Kent D.; Hirshleifer, David; } \\
\text { Subrahmanyam, Avanidhar }\end{array}$ \\
\hline 296 & Journal of Financial Economics & 1997 & $\begin{array}{l}\text { Detecting long-run abnormal stock re- } \\
\text { turns: The empirical power and specifi- } \\
\text { cation of test statistics }\end{array}$ & Barber, Brad M.; Lyon, John D. \\
\hline 291 & Journal of Financial Economics & 1996 & $\begin{array}{l}\text { Higher market valuation of companies } \\
\text { with a small board of directors }\end{array}$ & Yermack, David L. \\
\hline 271 & Journal of Financial Economics & 1998 & A model of investor sentiment & $\begin{array}{l}\text { Barberis, Nicholas; Shleifer, Andrei; } \\
\text { Vishny, Robert W. }\end{array}$ \\
\hline
\end{tabular}


Através da Tabela 2, pode-se perceber que os artigos mais citados foram publicados no Journal of Finance, corroborando a informação a respeito do fator de impacto (JCR). Outros periódicos também presentes na lista são o Journal of Financial Economics e o Mathematical Finance.

Quanto aos autores, destaca-se que Shleifer e Vishny são os autores mais citados da área de Finanças, uma vez que seu artigo foi citado 713 até o final de 2006.

Na base de dados do ISI, há a possibilidade de pesquisar quantidade de citações (ISIHighlyCited.com), uma vez que são disponibilizados os 250 pesquisadores mais citados das 21 áreas classificadas pelo ISI. Na área denominada Economic/Business, a qual engloba Finanças, os autores que são apresentados como altamente citados tanto pelo Scopus (Tabela 3) como pelo ISI são Shleifer, Vishny, Fama e French.

\section{Considerações Finais}

A análise de periódicos internacionais da área de Finanças, os quais estão disponíveis no Portal Periódicos CAPES, permitiu examinar as características desses journals e ainda apresentar artigos e autores mais citados da área estudada. Inicialmente, a amostra contava com 187 periódicos. Após algumas exclusões, chegou-se a um total de 72 periódicos que foram estudados.

A pesquisa objetivou investigar quais os periódicos estavam indexados ao Scopus, ISI e JCR, os países-sede dos periódicos, a freqüência com a qual são publicados (periodicidade), a existência de taxa de submissão e seu valor, taxa de corte, tempo para publicação após a aprovação de um artigo, índice de internacionalização do corpo editorial, autores e artigos mais citados.

A análise dos resultados demonstrou que 49 dos 72 periódicos analisados estão indexados à base de dados Scopus, 25 estão indexados ao ISI, apenas 18 periódicos publicaram em seus sites o fator de impacto, calculado pelo JCR, e 21 periódicos não estão indexados a nenhuma das bases de dados estudadas. Vale ressaltar que os periódicos que apresentaram maior impacto foram: Journal of Finance (2,549), Journal of Financial Economics $(2,385)$ e Review of Financial Studies $(1,893)$.

Quanto aos países dos periódicos, 51 (70,83\%) destes estão sediados nos Estados Unidos, demonstrando a hegemonia do país na área. Isso pode ser explicado pelo desenvolvimento da pesquisa em Finanças nesse país, além do crescente número de pesquisadores nas universidades americanas.

No que se refere à periodicidade dos journals analisados, 34 publicam edições trimestrais (quatro edições por ano). Além disso, há certa homogeneidade no que se refere à quantidade de periódicos que publicam três, cinco e seis edições por ano. 
Analisando a questão da cobrança da taxa de submissão, percebe-se um equilíbrio, uma vez que $51 \%$ dos periódicos fazem uso da taxa e, portanto, $49 \%$ não a cobram. Ainda com relação a essa característica, 13 periódicos cobram uma taxa de submissão entre US51eUS 100 e 11 periódicos cobram uma taxa de US\$ 40 a US\$ 50. Conseqüentemente, percebe-se que a maioria dos periódicos que cobram taxa de submissão, instituíram um valor de até US\$100. Quanto ao Brasil, os periódicos nacionais não cobram taxa de submissão.

Com base em respostas obtidas a partir dos editores-chefe dos journals, 14 periódicos demoram em torno de seis meses para publicar um artigo já aprovado. Por outro lado, periódicos como Finance India, Journal of International Financial Markets, Institutions and Money e Journal of Multinational Financial Management levam ao menos 15 meses para publicar um artigo já aprovado pelos avaliadores. Quanto à taxa de corte, outro item respondido pelo editores, a maioria dos periódicos aprova $20 \%$ ou menos dos artigos submetidos a eles. Nesta etapa, há restrição quanto aos resultados do estudo, uma vez que a análise foi baseada nas respostas dos editores. No entanto, no que se refere ao tempo para publicação, alguns periódicos já demonstram em seus artigos o intervalo de tempo entre submissão, aprovação e publicação final; e no que se refere à taxa de corte, certos periódicos apresentam, ao final de cada ano, um balanço final dos artigos publicados, informando a taxa de corte do período.

Outra restrição fundamental a ser mencionada é o fato de que, apesar de escolha ter recaído sobre periódicos que continham em seus títulos os termos finance ou financial, outros periódicos importantes da área de Administração, Contabilidade, Negócios e Economia também publicam artigos sobre Finanças. Contudo, essas revistas não foram analisadas como, por exemplo, Journal of Accounting \& Economics, Journal of Business e Econometrics, dentre outras.

A partir do cálculo da internacionalização do corpo editorial, percebeu-se que o periódico Research in International Business and Finance $(0,685)$ possui o corpo editorial mais internacionalizado na amostra de periódicos analisada, seguido do International Journal of Health Care Finance and Economics $(0,666)$ e do Financial History Review (0,562). Em contrapartida, os periódicos Journal of Applied Finance, Journal of Economics and Finance, Journal of Financial and Quantitative Analysis, Journal of Financial Economics e Research in Healthcare Financial Management apresentaram índice de internacionalização do corpo editorial nulo, demonstrando que possuem editores de somente um país.

E, finalmente, os cinco artigos mais citados foram publicados no Journal of Finance, sendo que o primeiro deles (autoria de Shleifer e Vishny (1997)) possuía 713 citações até dezembro de 2006. Outros periódicos também presentes na lista são o Journal of Financial Economics e o Mathematical Finance. Com relação aos autores, destaca-se que Shleifer, Vishny, Fama e French são apontados como autores da área de Finanças altamente citados no ISI. 


\section{Referências}

Baker, H. K. \& Mukherjee, T. K. (2007). Survey research in finance: Views from journal editors. International Journal of Managerial Finance, 3(1):11-25.

Borba, J. A. \& Murcia, F. D. (2006). Oportunidades para pesquisa e publicação em contabilidade: Um estudo preliminar sobre as revistas acadêmicas de língua inglesa do portal de periódicos da CAPES. Brazilian Business Review, 3(1):88103.

Borokhovich, K. A., Bricker, R. J., \& Simkins, B. J. (2000). An analysis of finance journal impact factors. Journal of Finance, 55(3):1457-1469.

Camargos, M. A., Coutinho, E. S., \& Amaral, H. F. (2005). O perfil da área de finanças do ENANPAD: Um levantamento da produção científica e de suas tendências entre 2000-2004. In ANPAD, editor, EnANPAD, 29, 2005, Curitiba. Anais, Brasília. CD-ROM.

Chan, K., Karolyl, G. A., \& Rhee, S. G. (2002). A retrospective evaluation of the Pacific-Basin finance journal: 1993-2002. Pacific-Basin Finance Journal, $10: 497-516$.

Chan, K. C., Chen, C. R., \& Steiner, T. L. (2004). Who is publishing? Ana analysis of finance research productivity in the European region. Journal of Business Finance and Accounting, 31(3):401-437.

Chan, K. C. \& Fok, R. C. W. (2003). Membership on editorial boards and finance department rankings. Journal of Financial Research, 26(3):405-420.

Fama, E. F. (1970). Efficient capital markets: A review of theory and empirical work. Journal of Finance, 25(2):383-417.

Frandsen, T. F. (2004). Journal interaction: A bibliometric analysis of economic journals. Journal of Documentation, 61(3):385-401.

Frezatti, F. \& Borba, J. A. (2000). Análise dos traços de tendência de uma amostra das revistas científicas da área de contabilidade publicadas na língua inglesa. Caderno de Estudos, 13(24):50-78.

Grable, J. E. (2006). Personel finance, financial planning, ans financial couseling publication renkings. Journal of Personel Finance, 5(1):68-78.

Haugen, R. A. (2000). Os Segredos Da Bolsa: Como Prever Resultados e Lucras Com Ações. Pearson Educação, São Paulo.

Leal, R. P. C., Oliveira, J., \& Soluri, A. F. (2003). Perfil da pesquisa em finanças no Brasil. Revista de Administração de Empresas, 43(1):91-104. 
Mesquita, R. (2006). Elaboração e aplicação de instrumentos para avaliação da base de dados Scopus. Perspectivas em Ciência da Informação, 11(2):187-205.

Modigliani, F. \& Miller, M. H. (1958). The cost of capital, corporate finance and the theory of investment. American Economic Review, 48(3):261-97.

Neuhauser, K. L. (2007). Survey research in finance. International Journal of Managerial Finance, 3(1):5-10.

Peng, M. W. \& Zhou, J. Q. (2006). Most cited articles and authors in global strategy research. Journal of International Management, 12:490-508.

Shleifer, A. \& Vishny, R. W. (1997). A survey of corporate governance. Journal of Finance, 52(2):737-783.

Testa, J. (1998). A base de dados ISI e seu processo de seleção de revistas. Ciência da Informação, 27(2):233-235.

Vilhena, V. \& Crestana, M. F. (2002). Produção científica: Critérios de avaliação de impacto. Revista da Associação Médica Brasileira, 48(1):1-25. 


\section{Apêndice 1}

\section{Periódicos Analisados Indexados ao Scopus, ISI e JCR}

\begin{tabular}{|c|c|c|c|}
\hline Periódicos & Scopus & ISI & JCR \\
\hline Abacus: A Journal of Accounting, Finance and Business Studies & $\mathrm{X}$ & $\mathrm{X}$ & \\
\hline Accounting and Finance & $\mathrm{X}$ & & \\
\hline Annals of Finance & $\mathrm{X}$ & & \\
\hline Asia Pacific Financial Markets & $\mathrm{X}$ & & \\
\hline Decisions in Economics and Finance: Journal of Apllied Mathematics & $\mathrm{X}$ & & \\
\hline Emerging Markets, Finance and Trade & $\mathrm{X}$ & $\mathrm{X}$ & 0,259 \\
\hline European Financial Management & & $\mathrm{X}$ & \\
\hline Finance and Stochastics & $\mathrm{X}$ & $\mathrm{X}$ & 1,429 \\
\hline Financial Analysts Journal & $\mathrm{X}$ & $\mathrm{X}$ & 0,542 \\
\hline Financial History Review & $\mathrm{X}$ & & \\
\hline Financial Management & $\mathrm{X}$ & $\mathrm{X}$ & 0,976 \\
\hline Financial Markets, Institutions and Instruments & $\mathrm{X}$ & & \\
\hline Global Finance Journal & $\mathrm{X}$ & & \\
\hline International Finance & $\mathrm{X}$ & & \\
\hline International Journal of Finance and Economics & $\mathrm{X}$ & $\mathrm{X}$ & 0,234 \\
\hline International Journal of Health Care Finance and Economics & $\mathrm{X}$ & & \\
\hline International Review of Economics and Finance & $\mathrm{X}$ & & \\
\hline International Review of Financial Analysis & $\bar{X}$ & & \\
\hline International Tax and Public Finance & $\mathrm{X}$ & $\mathrm{X}$ & 0,603 \\
\hline Journal of Accounting, Auditing and Finance & $\mathrm{X}$ & & \\
\hline Journal of Banking and Finance & $\mathrm{X}$ & $\mathrm{X}$ & 0,531 \\
\hline Journal of Business Finance and Accounting & $\mathrm{X}$ & $\mathrm{X}$ & \\
\hline Journal of Business Venturing: Entrepr. Fin., Innov. and Reg. Developm. & $\mathrm{X}$ & & 1,846 \\
\hline Journal of Corporate Finance & $\mathrm{X}$ & $\mathrm{X}$ & 0,883 \\
\hline Journal of Empirical Finance & $\mathrm{X}$ & & \\
\hline Journal of Finance & $\mathrm{X}$ & $\mathrm{X}$ & 2,549 \\
\hline Journal of Financial and Quantitative Analysis & $\bar{X}$ & $\mathrm{X}$ & 1 \\
\hline Journal of Financial Econometrics & $\mathrm{X}$ & & \\
\hline Journal of Financial Economics & $\mathrm{X}$ & $\mathrm{X}$ & 2,385 \\
\hline Journal of Financial Intermediation & $\mathrm{X}$ & $\mathrm{X}$ & 1,118 \\
\hline Journal of Financial Markets & $\mathrm{X}$ & $\mathrm{X}$ & 0,974 \\
\hline Journal of Financial Research & $\mathrm{X}$ & $\mathrm{X}$ & \\
\hline Journal of Financial Services Research & $\mathrm{X}$ & $\mathrm{X}$ & \\
\hline Journal of Financial Stability & $\mathrm{X}$ & & \\
\hline Journal of International Financial Markets, Institutions and Money & $\mathrm{X}$ & & \\
\hline Journal of International Money and Finance & $\mathrm{X}$ & $\mathrm{X}$ & 0,505 \\
\hline Journal of Multinational Financial Management & $\mathrm{X}$ & & \\
\hline Journal of Property Investment and Finance & $\mathrm{X}$ & & \\
\hline Journal of Real Estate Finance and Economics & $\mathrm{X}$ & $\mathrm{X}$ & 0,473 \\
\hline Managerial Finance & & $\mathrm{X}$ & \\
\hline Mathematical Finance & $\mathrm{X}$ & $\mathrm{X}$ & 1,345 \\
\hline North American Journal of Economics and Finance & $\mathrm{X}$ & & \\
\hline Pacific Basin Finance Journal & $\mathrm{X}$ & & \\
\hline Public Finance Review & $\mathrm{X}$ & $\mathrm{X}$ & \\
\hline Quarterly Review of Economics and Finance & $\mathrm{X}$ & $\mathrm{X}$ & \\
\hline Research in Healthcare Financial Management & $\mathrm{X}$ & & \\
\hline Research in International Business and Finance & $\mathrm{X}$ & & \\
\hline Review of Finance & $\mathrm{X}$ & & \\
\hline Review of Financial Economics & $\mathrm{X}$ & & \\
\hline Review of Financial Studies & $\mathrm{X}$ & $\mathrm{X}$ & 1,893 \\
\hline Review of Quantitative Finance and Accounting & $\mathrm{X}$ & & \\
\hline
\end{tabular}




\section{Apêndice 2}

\section{Periódicos Analisados sem Indexação ao Scopus e/ou ISI}

\begin{tabular}{l}
\hline \multicolumn{1}{c}{ Periódicos } \\
\hline Finance India \\
\hline Financial Accountability and Management \\
\hline Financial Markets and Portfolio Management \\
\hline Financial Review \\
\hline Financial Services Review \\
\hline International Journal of Intelligent Systems in Accounting, Finance and Management \\
\hline Journal of Applied Finance \\
\hline Journal of Economics and Finance \\
\hline Journal of Education Finance \\
\hline Journal of Emerging Market Finance \\
\hline Journal of Financial Crime \\
\hline Journal of Financial Service Professionals \\
\hline Journal of Financial Services Marketing \\
\hline Journal of Government Financial Management \\
\hline Journal of International Financial Management and Accounting \\
\hline Journal of Pension Economics and Finance \\
\hline Journal of Public Budgeting, Accounting and Financial Management \\
\hline Journal of Risk Finance \\
\hline Multinational Finance Journal \\
\hline Public Budgeting and Finance \\
\hline Review of Accounting and Finance \\
\hline
\end{tabular}




\section{Apêndice 3}

\section{Taxa de Corte, Tempo para Publicação e IICE}

\begin{tabular}{|c|c|c|c|}
\hline Periódicos & Taxa de corte & Tempo para publicação & IICE \\
\hline Abacus: A Journal of Acc., Finance and Business Studies & $78 \%$ & $*$ & 0,333 \\
\hline Accounting and Finance & $76 \%$ & $*$ & 0,119 \\
\hline Annals of Finance & $90 \%$ & 6 meses a 1 ano & 0,197 \\
\hline Asia Pacific Financial Markets & $80 \%$ & 1 ano & 0,298 \\
\hline Decisions in Economics and Finance & & & *** \\
\hline Emerging Markets, Finance and Trade & $85 \%$ & 1 ano & 0,34 \\
\hline European Financial Management & & & 0,46 \\
\hline Finance and Stochastics & $75 \%$ & 3 meses & 0,387 \\
\hline Finance India & $42 \%$ & 15 meses & 0,415 \\
\hline Financial Accountability and Management & & & 0,425 \\
\hline Financial Analysts Journal & $85 \%$ & 6 meses & 0,029 \\
\hline Financial History Review & & & 0,562 \\
\hline Financial Management & $90 \%$ & 6 meses & 0,033 \\
\hline Financial Markets and Portfolio Management & $70 \%$ & 3 a 6 meses & 0,266 \\
\hline Financial Markets, Institutions and Instruments & & & $* *$ \\
\hline Financial Review & $85 \%$ & $*$ & 0,076 \\
\hline Financial Services Review & & & 0,047 \\
\hline Global Finance Journal & & & 0,16 \\
\hline International Finance & $85 \%$ & 3 meses & 0,261 \\
\hline International Journal of Finance and Economics & & & 0,377 \\
\hline International Journal of Health Care Finance and Economics & $70 \%$ & 6 meses & 0,666 \\
\hline International Journal of Intell. Syst. in Acc., Fin. and Manag. & $60 \%$ & $*$ & 0,395 \\
\hline International Review of Economics and Finance & & & 0,127 \\
\hline International Review of Financial Analysis & $90 \%$ & $*$ & 0,379 \\
\hline International Tax and Public Finance & & & 0,433 \\
\hline Journal of Applied Finance & $90 \%$ & 6 meses & 0 \\
\hline Journal of Banking and Finance & & & 0,4 \\
\hline Journal of Business Finance and Accounting & $90 \%$ & $*$ & 0,17 \\
\hline Journal of Business Venturing & & & 0,251 \\
\hline Journal of Corporate Finance & $95 \%$ & 8 meses & 0,024 \\
\hline Journal of Economics and Finance & $80 \%$ & 1 ano & 0 \\
\hline Journal of Education Finance & & & *** \\
\hline Journal of Emerging Market Finance & & & 0,4 \\
\hline Journal of Empirical Finance & $65 \%$ & 6 meses & 0,226 \\
\hline Journal of Finance & $94 \%$ & 1 ano & 0,076 \\
\hline Journal of Financial and Quantitative Analysis & $92 \%$ & 1 ano e meio & 0 \\
\hline Journal of Financial Crime & & & 0,463 \\
\hline Journal of Financial Econometrics & $62 \%$ & 3 a 6 meses & 0,412 \\
\hline Journal of Financial Economics & & & 0 \\
\hline Journal of Financial Intermediation & $90 \%$ & 4 meses & 0,233 \\
\hline Journal of Financial Markets & $88 \%$ & 6 a 12 meses & 0,091 \\
\hline Journal of Financial Service Professionals & $60 \%$ & 3 a 6 meses & $* *$ \\
\hline Journal of Financial Services Marketing & $*$ & 3 meses & 0,105 \\
\hline Journal of Financial Services Research & & & 0,178 \\
\hline Journal of Financial Stability & $85 \%$ & Até 1 ano & 0,384 \\
\hline Journal of Government Financial Management & $50 \%$ & 3 a 6 meses & $* *$ \\
\hline Journal of International Financ. Manag. and Accounting & $79 \%$ & $*$ & 0,288 \\
\hline
\end{tabular}


Journal of International Financ. Markets, Instit. and Money $\quad 85 \% \quad 18$ meses $\quad 0,233$ \begin{tabular}{llll}
\hline Journal of International Money and Finance & $85 \%$ & 1 ano e meio & 0,347 \\
\hline
\end{tabular}

\begin{tabular}{llll}
\hline Journal of Multinational Financial Management & $85 \%$ & 18 meses & 0,331 \\
\hline
\end{tabular}

\begin{tabular}{lc}
\hline Journal of Pension Economics and Finance & 0,314 \\
\hline
\end{tabular}

\begin{tabular}{lc}
\hline Journal of Property Investment and Finance & 0,284
\end{tabular}

\begin{tabular}{lll}
\hline Journal of Public Budgeting, Acc. and Financ. Management $80 \%$ & $*$ & 0,084
\end{tabular}

\begin{tabular}{lc}
\hline Journal of Real Estate Finance and Economics & 0,162 \\
\hline
\end{tabular}

Journal of Risk Finance $\quad 75 \% 3$ meses e meio 0,144

\begin{tabular}{lc}
\hline Managerial Finance & 0,08 \\
\hline MathematicalFinance
\end{tabular}

\begin{tabular}{llll}
\hline Mathematical Finance & $94 \%$ & 1 ano & 0,37 \\
\hline
\end{tabular}

\begin{tabular}{lc}
\hline Multinational Finance Journal & 0,323 \\
\hline
\end{tabular}

\begin{tabular}{llll}
\hline North American Journal of Economics and Finance & $84 \%$ & 6 meses & 0,081 \\
\hline
\end{tabular}

\begin{tabular}{lc}
\hline Pacific Basin Finance Journal & 0,301 \\
\hline Pub*
\end{tabular}

\begin{tabular}{llll}
\hline Public Budgeting and Finance & & & \\
\hline Public Finance Review & $78 \%$ & 4 meses & 0,153 \\
\hline
\end{tabular}

\begin{tabular}{llll}
\hline Public Finance Review & $78 \%$ & 4 meses & 0,153 \\
\hline Quarterly Review of Economics and Finance & & & 0,072
\end{tabular}

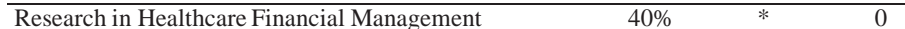

\begin{tabular}{llll}
\hline Research in International Business and Finance & $90 \%$ & 6 meses & 0,685 \\
\hline
\end{tabular}

\begin{tabular}{llll}
\hline Review of Accounting and Finance & $75 \%$ & $*$ & 0,226 \\
\hline
\end{tabular}

\begin{tabular}{lc}
\hline Review of Finance & 0,446 \\
\hline
\end{tabular}

\begin{tabular}{lr}
\hline Review of Financial Economics & 0,72 \\
\hline
\end{tabular}

\begin{tabular}{llll}
\hline Review of Financial Studies & $94 \%$ & 6 meses & 0,108 \\
\hline
\end{tabular}

\begin{tabular}{llll}
\hline Review of Quantitative Finance and Accounting & $85 \%$ & 1 ano & 0,069 \\
\hline
\end{tabular}

